\title{
Publicidad y traducción: el robo de mitos y contramitos en un mundo globalizado
}

\author{
María Calzada Pérez \\ Universidad Jaume I, Castellón
}

[...] mientras el consumismo se dedica a llenar nuestras necesidades más innecesarias [...] (Benedetti 1999: 141)

\begin{abstract}
Advertising is an essential component of a new form of postcolonialism globalization (and its associated partner consumerism). Some form of critical resistance seems necessary. Supporting the 'translation turn' (Bassnett 1998), the present paper wishes to underline the significance of translation studies for the analysis of textual rewritings in order to analyse textual rewritings (ads in this case). Drawing on Vidal Claramonte (1998) and semiotics (Barthes in particular), it discusses the notions of denotation, connotation, myth and countermyth and applies them to the study of three original and translated/translatable printed ads.
\end{abstract}

\section{Introducción}

El siglo XXI se abre con vivos, y a menudo airados, debates en torno al fenómeno de la 'globalización' (calco ya aceptado en castellano por una mayoría efectivamente 'globalizada') o la 'mundialización' (término ortodoxo con el que se enfrentan a diario los puristas de la lengua española a los correctores lingüísticos de los programas informáticos de Bill Gates). Dicho fenómeno, que vendría a ser una nueva forma de colonialismo inteligente, en esencia económico, derriba o construye (al gusto) fronteras nacionales, culturales, lingüísticas, textuales y hasta personales para apuntalar la barrera más subliminal y efectiva de la historia: la que separa al poder del resto de población; la que asila, en palabras polisistémicas, al núcleo más canónico de la periferia; la que, en definitiva, distancia y 'preserva' a los 'unos' de los 'otros'. Con ese fin, la globalización/mundialización recurre a todos los medios que tiene a su alcance y promueve otros de nuevo cuño. Se apropia de discursos 'progresistas y dinámicos'; se oculta tras palabras ambiguas o incluso vacías y desata una terrible ofensiva naturalizadora que nos convence de que sus propuestas son lógicas, normales y, a veces, hasta únicas. Porque la mundialización/globalización no se conforma con imponer su ideología (como conjunto de ideas que configuran nuestra percepción del mundo) ni con apoyar ciertos intereses frente a otros sino que, a su vez, se empeña en 'vencer convenciendo'.

Pero junto con la globalización/mundialización/colonialismo actuales surgen movimientos de resistencia antiglobalizadores/antimundializadores/poscoloniales. Los antiglobalizadores se proponen destruir - quizá des- 
construir y ¿reconstruir? - la norma mundializadora. Por tanto, es lógico que con frecuencia se pertrechen, por ejemplo, de las útiles herramientas de reflexión y crítica que ofrecen corrientes de pensamiento como el postestructuralismo, el poscolonialismo y la desconstrucción, cuyo objetivo no es otro que el de cuestionar, o descontruir, las propuestas naturalizadas de, entre otros, el estructuralismo y el colonialismo anteriores. Con cierta cautela, conviene, no obstante, recordar que dichas corrientes de pensamiento han sido objeto de usos ideológicos distintos y hasta opuestos (conservadores y progresistas), como refleja Gentzler (2002: 197). Es también notorio que han recibido críticas perspicaces (véase Tymoczko 1999; Robinson 1997 y Gentzler 2002 sobre el marco 'de resistencia' venutiano).

Por lo demás, parece oportuno argüir que al debate globalizador/antiglobalizador le ha llegado el momento del 'translation turn' (Bassnett 1998). Es decir, si - a la hora de estudiar fenómenos traductores varios - los estudios de traducción han de depurar sus metodologías y planteamientos surtiéndose de corrientes intradisciplinares e interdisciplinares, la misma traductología puede y debe convertirse en herramienta básica de otras disciplinas que ahora también se aproximan a la globalización, mundialización y colonialismo. Y ello por razones muy diversas. Citaremos aquí, por restricciones de espacio, tan sólo una de estas razones, que nos parece pertinente por el tema que nos ocupa. Los estudios traductológicos pueden, en concreto, arrojar información sobre el mundo posmoderno contemporáneo porque, como argumenta Apter (2001), la globalización fabrica productos caracterizados por una traducibilidad (anglo-americana) inherente. Es decir, todo aquello que se lanza al mercado mundial, ya antes de su elaboración, se diseña para que pueda traducirse, entenderse y, sobre todo, consumirse en inglés. Si la globalización, por tanto, desencadena mecanismos traductores incluso en artefactos monolingües, entonces la utilidad de los estudios de traducción parece incontestable.

En consecuencia, el presente artículo aspira a participar en el debate globalizador desde el terreno de la traductología. Ello supone un respaldo explícito a la exportación metodológica por la que aboga el 'translation turn'. La experiencia que nuestra disciplina posee para examinar, valorar, (re)producir mensajes; para indagar en las consecuencias ideológicas del intercambio multicultural; y para desenmascarar a las instituciones emisoras de dichos mensajes desde posicionamientos poscoloniales de tipos distintos (contra el colonialismo nacional, cultural, genérico, ...) hacen, creo, de los estudios de traducción un interlocutor valioso en el debate. Vaya por delante que este estudio asume cierto grado de parcialidad (ninguna reflexión crítica es totalmente objetiva). Con otras propuestas (como la de Beasly \& Danesi 2002), alimenta, desde un prisma afin al postestructuralismo y al poscolonialismo, la crítica y la resistencia y hace frente, en la medida de lo posible, a mecanismos de manipulación globalizadora. En definitiva, hago aquí míos los propósitos 'semioclastas' de Barthes: "No denunciation without an appropriate method of detailed analysis, no semiology which cannot, in the last analysis, be acknowledged as semioclasm"(1972: 9). 
Por último, en las presentes páginas, la traductología se adentra en el terreno de la publicidad, uno de los ámbitos donde la naturalización globalizadora se hace más exacerbada. Tras una breve introducción al fenómeno publicitario (apartado 1), se presenta (en el apartado 2) el enfoque híbrido (inter e intradisciplinar) con el que se analiza un número limitado de anuncios. Dicho análisis se centra en conceptos semióticos de profundo calado: la denotación, la connotación, el mito y el contramito (apartados 2.2.1, 2.2.2. y 2.2.3). Con todos ellos, pretendemos poner en evidencia alguno de los mecanismos que utiliza la naturalización globalizadora. No en vano, los anuncios son portavoces de uno de los mitos más poderosos de la actualidad: la sociedad de consumo. Convencidos estamos de que la convivencia cotidiana ha de organizarse en torno a sociedades de consumo en las que la identidad de sus miembros se define no por lo que éstos son ni por lo que conocen o saben, sino por lo que tienen o mejor por lo que compran. Convencidos seguimos de que (cierto) poder adquisitivo es equiparable al poder real, al poder de facto. Y que dicho poder 'nos hace libres' cuando, en definitiva, tan sólo nos hace libres para seguir comprando todo aquello que se nos vende con el objeto de tenernos entretenidos y desviar nuestras miradas de la barrera más subliminal y efectiva de la historia: la que nos separa a los 'otros' de 'los unos'.

\section{La publicidad}

Allá donde mira un occidental (casi siempre globalizado) su vista tropieza de sopetón, y sin buscarlo, con un anuncio. Frédéric Beigbeder, publicista retirado por iniciativa propia y actual escritor de best-sellers - como 13, 99 euros - sobre el mundillo de los anuncios explica que:

El ojo humano nunca había estado tan solicitado en toda su historia: se calculaba que entre el nacimiento y los dieciocho años, cualquier persona estaba expuesta a una media de 350.000 anuncios [...] Según el estudio antes citado, el occidental medio era sometido a 4000 mensajes comerciales diarios (Beigbeder 2000: 56-57).

Han transcurrido ya siglos desde que, según White (en Cook 2001: 6), aquel burdel de Éfeso se anunciara por vez primera en la historia. Ahora la ubicuidad publicitaria es tal que viaja en nuestros medios de transporte más públicos, invade nuestras neveras (adoptando, entre otras, la forma de decorativos imanes) y se cuela en nuestros hogares a través de la pequeña pantalla. No le falta razón a Klein (2001) cuando nos grita que el producto televisivo es una sucesión de anuncios que se interrumpe con películas, telediarios, etc.

No nos engañemos, Angela Goddard tiene toda la razón cuando advierte: "Make no mistake: Advertising works" (1998: 2). La publicidad constituye, sin sombra de dudas, una de las industrias más pujantes del pasado siglo XX y posee la virtud de adaptarse a las metaformosis que le requie- 
ren los nuevos tiempos. Funciona de manera 'abierta' y 'encubierta'. Dicho de otro modo, su mensaje nos perfora el cerebro con taladros explícitos e implícitos, que en este caso son casi siempre subliminales. ¿Conviene hacer algo?

La publicidad es motivo de estudio (a veces con entusiasmo, otras con preocupación) para la más variopinta gama de disciplinas. Publicitarios de reconocido prestigio (Aitchison 1999; Robinson 2000), analistas de divulgación (Beigbeder 2000; Klein 2001), autores de novelas exquisitas (Gopegui 2001), juristas o iniciados en la materia legal (Zambrana 2002; Martín et al. 1996) entre tantos otros se han aproximado al producto y al proceso publicitario. Abogando, como se ha visto arriba, por el 'translation turn', ¿por qué no hacerlo desde la traductología?

$\mathrm{Si}$ en algo coinciden, a grandes rasgos, todos los enfoques sobre publicidad citados en las referencias bibliográficas es en la caracterización que se hace de la misma. Bueno parece destilar el sentir general cuando, tras recoger opiniones diversas, emite su propia definición del fenómeno publicitario: "Publicidad es un tipo de comunicación que pretende decidir o cambiar la opinión sobre las cosas y la acción de sus receptores por medio de unas técnicas que actúan sobre el intelecto" (2000: 28). A esto cabría añadir los matices que resaltan Beasley \& Danesi, quienes subrayan, fundamentalmente, el carácter naturalizador del género publicitario, que convierte ciertas prácticas en rutinarias mientras abandona o soslaya otras (no por ello menos naturales per se):

We refer to advertising as a form of discourse in the sense that it has influenced not only the structure of language and modality of lifestyle, but also the content of routine daily acts of communicative exchanges. (2002: 16)

Por lo que concierne a la tipología de subgéneros publicitarios, son de nuevo, numerosas las propuestas académicas (por ejemplo, Al Shehari 2001; Bueno 2000; Goddard 1998). No obstante, la tipología publicitaria más habitual, la más clara y también la más concisa distingue entre anuncios dependiendo del canal en el que éstos aparecen: prensa, radio, televisión, internet. En el apartado 2.2. se examinan productos exclusivamente impresos. La razón es sencilla: tras una reflexión meditada, ha parecido más práctico depender de un material que, en principio, plantea menos escollos técnicos y que facilita la exposición académica del material que se examina. Los anuncios impresos son de fácil consulta para el lector, lo cual facilita el seguimiento de las reflexiones sobre los componentes lingüísticos y visuales del anuncio. Eso no es posible con mensajes publicitarios difundidos por otros medios.

Sin embargo, antes de embarcarnos en el examen práctico de los anuncios estudiados en este artículo conviene presentar, primero (en el apartado 2), el enfoque metodológico con el que se realizará el análisis posterior (apartados 2.2.1., 2.2.2. y 2.2.3.), acudiendo entre otros a Barthes, una de las principales fuentes de inspiración de este trabajo. 


\section{Metodología de análisis y aplicación}

De entre las numerosas aproximaciones exegéticas a la actividad traductora, Vidal Claramonte (1998) propone el siguiente modelo apoyándose en la obra de Foucault y en el pensamiento de traductólogas feministas, en su mayoría canadienses (Vidal Claramonte 1998: 101-120):

\begin{tabular}{|l|l|l|}
\hline $\begin{array}{l}\text { ARQUEOLOGÍA } \\
\text { DEL SABER }\end{array}$ & $\begin{array}{l}\text { GENEALOGÍA } \\
\text { DEL PODER }\end{array}$ & ACTUACIÓN (ÉTICA) \\
DESCRIPCIÓN & $\begin{array}{l}\text { EXPLICACIÓN/ } \\
\text { INTERPRETACIÓN }\end{array}$ & PRÁCTICA DE \\
TRADUCCIÓN \\
DE FACTORES & \\
& $\begin{array}{l}\text { ILOCUTIVOS } \\
\text { (INTENCIONES) }\end{array}$ & \\
& Y PERLOCUTIVOS & \\
& (EFECTOS) & \\
\hline
\end{tabular}

Para Vidal Claramonte, el análisis de textos traducidos (o traducibles) habría de pasar por tres etapas. En la primera - la arqueología del saber - el estudioso describe los rasgos 'texturales' o superficiales del texto. Obtiene, así, una visión general de la naturaleza y disposición de los elementos visibles del mismo. En la segunda etapa - la genealogía del poder -, el estudioso profundiza en su análisis y ofrece una explicación crítica (de talante socio-político) a las descripciones anteriores. La tercera etapa - la actuación - es el resultado lógico de descripción y explicación. El traductólogo adopta posturas (éticas) mediante las que apoya o subvierte el sistema establecido (y naturalizado). La combinación de descripción y explicación para apuntalar posicionamientos vitales ante las prácticas globalizadoras que nos circundan viene también auspiciada, fuera del ámbito traductológico, por teóricos de renombre como Barthes: "And yet this is what we must seek: a reconciliation between reality and men, between description and explanation, between object and knowledge" (Barthes 1972: 158).

Conviene, como siempre, ser cautos en nuestros estudios e insistir en que éste es sólo uno de los numerosos enfoques que pueden utilizarse para examinar el trasvase, en este caso, de textos publicitarios. Soy consciente de que se quedarán muchas cosas en el tintero, pero pienso, con Cook, que hay algo de positivo en esta "deficiencia":

Discourse - especially discourse as complex as advertising - always holds out more to be analysed, leaves more to be said. But this need not be a cause of despair. It would be both depressing and self deceptive to believe that one could exhaust all the aspects of the genre, and present an answer to all the problems it poses. (2001: 5) 


\subsection{Descripción de la publicidad}

La descripción de la publicidad y su vinculación con la traductología han sido objeto de estudio minucioso por los trabajos de teóricos como Bueno (2000) y Elena $(1990,1994,2001)$. Esta última aborda en sus manuales los rasgos macro y microestructurales (verbales y no verbales) de la publicidad así como las herramientas útiles para trasladar los textos publicitarios al castellano. Por su parte, Bueno (2000) se detiene, entre otros aspectos, en la composición del texto publicitario (marca, logotipo, eslogan, mensaje) y presenta un laborioso estudio sobre la retórica, la publicidad y la práctica de la traducción. Asimismo analiza la situación comunicativa en la que se intercambia el mensaje publicitario (con sus funciones y participantes). Por razones de espacio, no obstante, el presente artículo se centra en la etapa explicativa del marco propuesto, que aborda los aspectos ideológicos vinculados a algunos mecanismos que favorecen la naturalización globalizadora y la cultura del consumismo. Para profundizar en la fase descriptiva, remitimos, entre otros, a los dos estudios mencionados aquí.

\subsection{Explicación de la publicidad}

A nadie se le escapa que la publicidad (como el resto de los géneros textuales) no se queda en el nivel de la descripción. Va más allá y produce efectos colaterales que encuentran sus causas en razonamientos ilocutivos y provoca efectos claramente perlocutivos. ¿Qué mayor perlocución que salir de casa, entrar en un punto de venta y adquirir un producto del que el consumidor no tiene necesidad sólo porque nuestra sociedad (a través del bombardeo mediático) le incita a hacerlo? La explicación de causas ilocutivas y efectos perlocutivos parece insoslayable si, como Barthes, nos atrevemos no sólo a destapar sino incluso a cuestionar el consumismo naturalizado contemporáneo:

I had just read Saussure and as a result acquired the conviction that by treating 'collective representations' as sign-systems, one might hope to go further than the pious show of unmasking them and account in detail for the mystification which transforms petit-bourgeois culture into a universal culture. (1972: 9)

Para remontarnos a causas ilocutivas y efectos perlocutivos pueden escogerse caminos diversos, todos ellos apropiados. Se me ocurren en estos momentos como mínimo tres. La teoría del polisistema divulgada entre otros por Itamar Even-Zohar. El Análisis Crítico del Discurso (CDA) abanderado por Fairclough; Fowler; Kress etc. Y la semiótica. En este artículo considero oportuno adentrarme por la vía semiótica (como también hice en Vidal Claramonte y Calzada Pérez en prensa) ya que, al igual que Hatim, "I take the supremacy of the semiotic dimension to be indubitable" (1996: 117). La 
fusión de semiótica y publicidad no es una práctica académica extendida como explican Beasly \& Danesi cuando dicen "And to the best of our knowledge, few textbook treatments of advertising from the semiotic perspective - if any - are currently available" (2002: v). A su vez, los encuentros entre semiótica y traducción son más bien incipientes. De estos encuentros se desprenden estudios como, por ejemplo, Jakobson, Osimo (2001), Gorlee (1994) o Al Shehari (2001). Sin embargo, la combinación inter e intradisciplinar - traducción, semiótica y publicidad - ofrece un potencial inmenso para abordar la complejidad de los fenómenos traductológicos mediante herramientas relativamente sencillas.

Stam et al. definen la semiótica como "the study of signs, signification and signifying systems"(1992: 1). Esta definición es especialmente esclarecedora para organizar estudios críticos, de inspiración semiótica, en el seno de la traductología. Así, el investigador puede analizar el funcionamiento más básico del signo (combinación de significante y significado que establece relaciones internas y externas: paradigmáticas y sintagmáticas). Éste es el objeto de estudio de Vidal Claramonte y Calzada Pérez (en prensa). También puede concentrarse en el entramado que crean los sistemas significativos y focalizar la atención en la intertextualidad (Calzada Pérez en prensa). Finalmente, el estudioso puede profundizar en el concepto de significación (en sus diferentes niveles de denotación, connotación, mito y contramito) de la mano imprescindible de Barthes. Éste es el propósito del presente trabajo.

\subsubsection{Denotación y connotación en anuncios originales y traducidos}

Denotación, connotación, mito y contramito. Conceptos esenciales en la teoría barthiana. Expliquémoslos poco a poco con anuncios relevantes que pueden afectar a la labor del traductor.

Como es sabido, la denotación recoge, según Barthes, de forma muy simplificada, el significado 'literal' y 'obvio' y de las expresiones tanto verbales como visuales. La connotación, en cambio, se refiere a las asociaciones socioculturales y personales que van adquiriendo esos significados. Como inciso, diré que el mismo Barthes nos alerta de que esta dicotomía puede convertirse en reduccionista y hasta peligrosa: "Denotation is not the first meaning, but pretends to be so; under this illusion, it is ultimately no more than the last of connotations" (1974: 9). Quede claro que también yo opino que las denotaciones de nuestras sociedades no son, por regla general, más que connotaciones naturalizadas y aceptadas como verdades inamovibles que imponen los núcleos de poder.

La denotación, en definitiva, se representaría así:

\begin{tabular}{|l|l|}
\hline 1. SIGNIFICANTE & 2. SIGNIFICADO \\
\hline \multicolumn{3}{|c|}{ 3. SIGNO (DENOTACIÓN) } \\
\hline
\end{tabular}


La connotación, sin embargo, funcionaría, según Barthes (1972: 115) de este otro modo:

\begin{tabular}{|l|l|}
\hline 1. SIGNIFICANTE & 2. SIGNIFICADO \\
\hline 3. SIGNO /SIGNIFICANTE [FORMA] & 4. SIGNIFICADO [CONCEPTO] \\
\hline \multicolumn{2}{|c|}{ 5. SIGNIFICACIÓN } \\
\hline
\end{tabular}

Cabe destacar que, para Barthes (1972: 122), la naturaleza de la significación (casilla 5) que surge del encuentro entre forma (casilla 3) y concepto (casilla 4) es de deformación o distorsión, lo cual es lógico porque el concepto siempre distorsiona relaciones significativas de niveles anteriores. Esta significación/distorsión nunca es arbitraria y persigue propósitos evidentes a veces implícitos y otras explícitos.

Ahora que ya se ha recordado el funcionamiento de denotación y connotación trasladémonos al mundo publicitario y a su repercusión en la práctica traductora. El siguiente anuncio de la conocida marca de artículos electrónicos Bang \& Olufsen es a la vez texto original y traducido que, sin cambio alguno, se orienta tanto al mercado anglosajón como al hispano. Estudiamos sobre el original los niveles denotativo y connotativo del mismo.

La denotación del anuncio vendría a ser así:

\begin{tabular}{|c|c|}
\hline $\begin{array}{l}\text { 1. Objetos estilizados de colores } \\
\text { grisáceos y verdosos }\end{array}$ & 2. Televisor \\
\hline $\begin{array}{l}\text { Confusión policromática e } \\
\text { indefinición de la imagen } \\
\text { de fondo }\end{array}$ & Ambiente borroso, 'entre nieblas' \\
\hline $\mathrm{S} / \mathrm{t} / \mathrm{a} / \mathrm{t} / \mathrm{u} / \mathrm{e} \mathrm{o} / \mathrm{f} / \mathrm{l} / \mathrm{i} / \mathrm{b} / \mathrm{e} / \mathrm{r} / \mathrm{t} / \mathrm{y}$ & Statue of liberty \\
\hline \multicolumn{2}{|l|}{ 3. Televisor de Bang \& Olufsen } \\
\hline \multicolumn{2}{|c|}{$\begin{array}{l}\text { Monumento de fama mundial que se encuentra en Nueva York y } \\
\text { que Francia regaló a EE UU }\end{array}$} \\
\hline
\end{tabular}




\section{STATUE OF LIBERTY}

Anuncio 1: Texto original y traducido de un anuncio de Bang \& Olufsen 
La connotación, por el contrario, la reflejaríamos del modo siguiente:

\begin{tabular}{|c|c|}
\hline $\begin{array}{l}\text { 1. Objetos estilizados de colores } \\
\text { grisáceos y verdosos }\end{array}$ & 2. Televisor \\
\hline $\begin{array}{l}\text { Confusión policromática e } \\
\text { indefinición de la imagen } \\
\text { de fondo }\end{array}$ & Ambiente borroso, 'entre nieblas' \\
\hline $\mathrm{S} / \mathrm{t} / \mathrm{a} / \mathrm{t} / \mathrm{u} / \mathrm{e} \mathrm{o} / \mathrm{f} / \mathrm{l} / \mathrm{i} / \mathrm{b} / \mathrm{e} / \mathrm{r} / \mathrm{t} / \mathrm{y}$ & Statue of liberty \\
\hline 3. Televisión de Bang \& Olufsen & 4. + 'Americanicity' \\
\hline $\begin{array}{l}\text { Monumento de fama mundial } \\
\text { que se encuentra en Nueva York } \\
\text { y que Francia regaló a EE UU }\end{array}$ & $\begin{array}{l}\text { ['Americanicidad'] (término } \\
\text { que hemos creado por analogía } \\
\text { con "Italianicity", el vocablo que } \\
\text { utilizó Barthes al analizar un } \\
\text { anuncio de pasta italiana). } \\
\text { En el mundo anglosajón: valores } \\
\text { de independencia, libertad, demo- } \\
\text { cracia, posibilidades para todos } \\
\text { progreso, etc. }\end{array}$ \\
\hline \multicolumn{2}{|c|}{$\begin{array}{l}\text { 5. El televisor Bang \& Olufsen es significación de la 'Americanicity' y } \\
\text { todos los valores que este término suscita. En el mundo anglosajón este } \\
\text { término suele ser positivo y ligarse a otros como: la independencia, la } \\
\text { libertad, la democracia, las posibilidades para todos, el progreso. El anun- } \\
\text { cio se convierte en un símbolo. El comprador de una Bang \& Olufsen } \\
\text { busca ese 'estilo de vida americano', que en este mundo algo confuso } \\
\text { ('entre nieblas') tan sólo nos puede traer la susodicha tele (sola ante una } \\
\text { atmósfera de 'nada'). La Bang \& Olufsen aportará todas estas cualidades } \\
\text { a nuestras vidas anodinas. }\end{array}$} \\
\hline
\end{tabular}

Queda por tanto claro que original y traducción coinciden exactamente en el primer plano de los significantes. Y sin embargo, conforme vamos recorriendo la cadena de significación crecen las diferencias entre un texto redactado para el mercado angloparlante y el orientado a los consumidores hispanos. El concepto artificial de la 'Americanicity' (casilla 4) puede crear asociaciones muy diversas en ambos entornos y el resultado sería connotaciones dispares (y hasta encontradas) que se traducirían en comportamientos distintos de los consumidores de habla (y cultura) inglesa o española. Para un consumidor estadounidense, como se muestra en la tabla, la 'Americanicity' despliega connotaciones siempre positivas (ligadas a conceptos de libertad, independencia, democracia... anglosajones) y le anima a comprar el producto. Para un consumidor hispano, las asociaciones de la 'Americanicity' no son necesariamente positivas y pueden desestabilizar el 
éxito del producto. En el espacio hispano (léase Latinoamérica principalmente) la 'Americanicity' ha resultado a menudo sangrienta y ha dejado víctimas (económicas, políticas, personales) en el camino. En términos venutianos, la estrategia de trasvase de este anuncio traducido (reproducción exacta de los significantes del original) sería una extranjerización incuestionable que, como extranjerización según Venuti, debería instar a la subversión y cuestionar la norma imperante. Y sin embargo, la extranjerización venutiana del anuncio traducido de Bang \& Olufsen no sólo mantiene las connotaciones (colonizadoras) del mundo anglosajón, despreciando el resto de asociaciones que podrían aflorar en el ámbito hispano, sino que además pone en peligro el objetivo más pragmático del texto publicitario: vender un televisor Bang \& Olufsen y potenciar, a fin de cuentas, la sociedad de consumo contemporánea.

\subsubsection{Mitos publicitarios y traducción}

La maquinaria de la significación no se detiene en los peldaños de denotación y connotación. El siguiente nivel es el mito que, en palabras de Barthes (1972: 129), "tranforms history into nature". El mito supone la naturalización de connotaciones cuyo impacto, en principio, sería más limitado: "What causes mythical speech to be uttered is perfectly explicit, but it is immediately frozen into something natural; it is not read as a motive, but as a reason" (Barthes 1972: 129).

Para Barthes (1972: 131) todo mito supone "a language robbery" (es decir, un robo de toda clase de lengua puesto que nada escapa a sus garras). Y eso porque su existencia supone la erradicación de significados anteriores y la imposición de otros nuevos cual si fueran universales. Todo ello con unos propósitos socio-económicos determinados.

Nadie puede negar que Marilyn Monroe ya es un gran mito de nuestra cultural universal/globalizada. Y los publicitarios lo saben. 


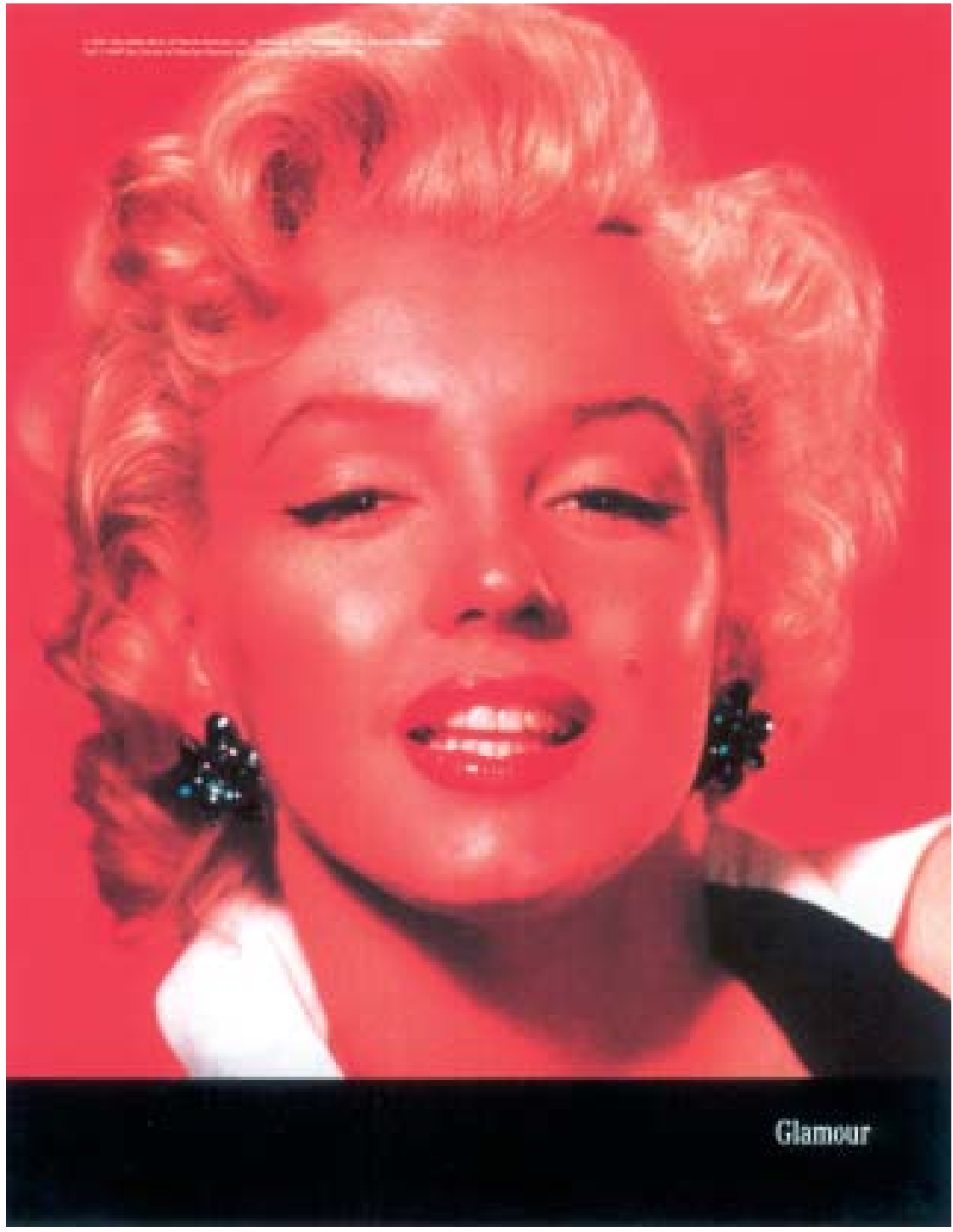

Anuncio 2: Texto original de un anuncio de Mercedes-Benz. 
Mercedes-Benz apoya la venta de su marca en un anuncio que se construye sobre el mito de la Monroe:

\begin{tabular}{|l|l|}
\hline $\begin{array}{l}\text { 1. Foto de una mujer rubia sobre } \\
\text { fondo rojo y pie negro }\end{array}$ & 2. Norma Jean Baker Morteson \\
\hline G/l/a/m/o/u/r & Glamour \\
\hline 3. Marilyn Monroe & $\begin{array}{l}4 .+ \text { actriz de cine + atractiva } \\
+ \text { recuerdo de sus películas, etc. }\end{array}$ \\
\hline $\begin{array}{l}\text { 5. Mujer glamourosa y sensual, representante de todas las mujeres atrac- } \\
\text { tivas. }\end{array}$ \\
$\begin{array}{l}\text { 'Mito erótico' (según el Diccionario enciclopédico Espasa) } \\
\text { (reforzado por la palabra glamour en blanco sobre fondo negro - el color } \\
\text { de la elegancia - y resaltado por el rojo, mito a su vez del erotismo y la } \\
\text { pasión. }\end{array}$ \\
\hline
\end{tabular}

El anuncio 2 no muestra ningún producto. En un pie (que de hecho se ubica en la parte superior izquierda de la página) en letras minúsculas puede leerse, con verdadera dificultad, el copyright del anuncio:

๑ 1997 Mercedes-Benz of North America, Inc.Montvale NJ [...]

${ }^{\circledR} 1997$ the Estate of Marilyn Monroe by CMGWorldwide Inc., Indpls, IN.

Pero pocos acercarán tanto la vista como para leer ese texto. En la parte inferior izquierda, el titular 'Glamour'. Mercedes-Benz es, piensan los publicistas, un mito de glamour comparable a Monroe. Tan reconocido es su prestigio que ni siquiera tiene que mostrar sus coches al consumidor. El único rastro de la firma alemana es el famoso lunar de la actriz, convertido ahora en el logotipo de la Mercedes. En definitiva, al vender sus productos, Mercedes-Benz vende el mito erótico de Marilyin Monroe. Y viceversa: Monroe es un señuelo que apela a los instintos del receptor para incitarlo a consumir.

La traducción al español no plantea mayores problemas: los mitos de Monroe y Mercedes son también conocidos en nuestro país. Nos sirven de ejemplo para ilustrar el funcionamiento del mito. Sin embargo ni publicistas ni traductores deberían pasar por alto que el mito de Monroe no sólo aglutina glamour, sensualidad y sexualidad sino que también, como sostiene Chandler (2001) se construye sobre la depresión, la ingerencia de drogas, narcóticos y alcohol y, en último término, la muerte. Los mitos, como el de Monroe, no son únicos e inamovibles. Se deslizan y, a veces, sus efectos son insospechados. El publicista (que es traductor a fin de cuentas) y el traductor (en estos casos publicista en el fondo) deben tener esta inestabilidad en 
cuenta y dar la voz de alarma para que ellos mismos u otros valoren los riesgos.

\subsubsection{Contramitos publicitarios y traducción}

Tampoco el mito pone el punto final al entramado significativo. Tras el mito se esconde la posibilidad del contramito. Porque como arguye Barthes, "Since myth robs language of something, why not rob myth?"(1972: 135). Ello supone que con los contramitos se desconstruyen mitos anteriores más o menos universales y a veces hasta centenarios con los que nos asociamos de manera consciente o inconsciente. La desconstrucción acometida por el contramito supone apropiarse de la significación universal del mito pero deslizarla de modo que cumpla con nuevos (y hasta encontrados) propósitos. La publicidad utiliza el contramito con pericia para vendernos productos y apelar, al mismo tiempo, a nuestros sentimientos arraigados. Pero en el actual mundo de globalización, en donde las campañas de publicidad, como indicaba Apter (2001), han de contemplar desde el primer momento la traducibilidad de los anuncios, cabe preguntarse cómo se trasvasan de una cultura a otra los contramitos para cumplir de manera efectiva el propósito del anunciante. Conviene asimismo plantearse si el traductor debe reproducir contramitos que atenten contra sus posicionamientos éticos; e incluso si puede legítimamente crear sus propios contramitos a partir de los contramitos originales para desestabilizar ideologías con las que no está de acuerdo.

El siguiente anuncio de Guinness se apoya sobre un inteligente contramito:

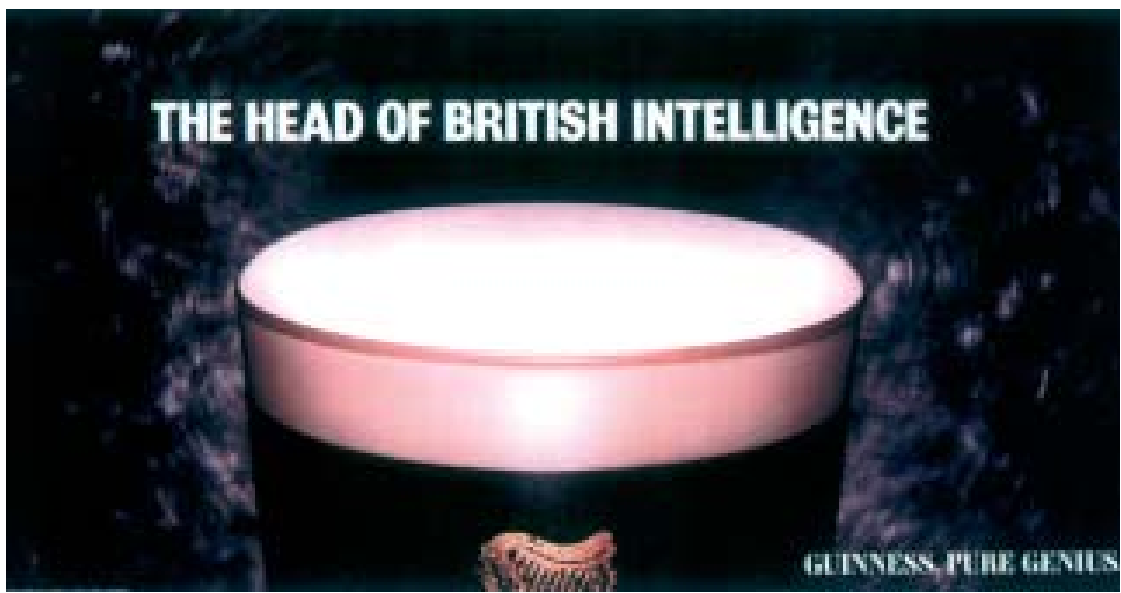

Anuncio 3: Texto original de un anuncio de Guinness

Sobre un fondo negro de distintas texturas, la parte superior de una pinta de Guinness fría atrae la mirada del consumidor. La espuma de la bebida es 
especialmente apetecible. En el vaso se aprecia el logotipo de la firma, cortado por la mitad (es tan conocido el logotipo de Guinness que sólo con insinuarlo le basta a la empresa para que el lector lo reconozca). Al pie del anuncio, el eslogan que creó - a mediados de la década de las ochenta - la agencia estadounidense Ogilvy \& Matter: "Guinness. Pure Genius". El titular en blanco reza así: "The head of British intelligence" (La cabeza/cúspide/cúpula de la inteligencia británica). Es precisamente el titular el foco de nuestro análisis desmitificador. Su denotación significativa es relativamente normal, como normal es que una 'cabeza' sea 'inteligente'. Las connotaciones de la leyenda sorprenden algo más puesto que el término 'head' en inglés se utiliza, en un segundo nivel de significación, para referirse a la espuma de la cerveza. Pero es la combinación de significante/significado/signo, denotaciones y connotaciones... la que activa, en este caso, un contramito con el que se ataca una mitología tan milenaria como la británica.

Si el consumidor está en contacto con la cultura del Reino Unido, no le será difícil reconocer el mito por el cual el pueblo británico convierte al irlandés en el objeto de todas sus burlas. Dicho de otro modo, los irlandeses son para los ingleses (y digo bien aquí, para los ingleses) lo que el mito de Lepe sugiere para los españoles. En este anuncio, Ogilvy \& Matter subvierte por completo esta mitología hasta tal punto que se sitúa un producto irlandés en la cúpula de la inteligencia británica. Y con la ayuda de una simple metonimia, se construye el contramito de que Irlanda supera al Reino Unido en inteligencia. Es más, se transmite el mensaje de que Irlanda lidera la inteligencia británica. El trasvase multicultural de toda esta tupida red ideológica tiene difícil solución si se desea traducirla al castellano. ¿Cuáles serían el éxito y las repercusiones ideológicas del empleo de estrategias extranjerizantes en la traducción de este anuncio? ¿Se transmitiría el mensaje de subversión socio-política que, en el Reino Unido e Irlanda, perciben los consumidores locales? ¿Sería posible una domesticación que permitiera mantener la imagen y al mismo tiempo resultara comprensible, a todos los niveles, para el consumidor hispano?¿Cuál sería el impacto ideológico de dicha domesticación? Y ¿qué se esconde realmente bajo el contramito subversivo que propone Guinness? El traductor de nuestro siglo XXI no puede dejar sin respuesta preguntas como éstas, que lo enfrentan a los mitos y contramitos de culturas foráneas.

\section{Conclusión}

Este artículo participa, desde la traductología inter e intradisciplinar, en el debate contemporáneo sobre la naturalización globalizadora y fenómenos (artificiales) como el consumismo actual, directamente asociado con ella. Así, me he detenido en el estudio de los conceptos de denotación, connotación, mito y contramito y he ilustrado su funcionamiento en anuncios originales y traducidos (Bang \& Olufsen) o con potencial de traducibilidad (Mercedes-Benz y Guinness). El examen realizado ha descrito los compo- 
nentes más superficiales de los anuncios (denotación) y se ha adentrado por terreno explicativo (connotación, mito y contramito). El objetivo último que se persigue aquí consiste en despertar en traductores (y demás consumidores) reflexiones éticas que afecten a sus trabajos y a sus vidas.

En concreto, tras el análisis de denotación, connotación, mito y contramito, el traductor percibe que las estrategias de resistencia venutianas (extranjerización) son mucho menos fiables de los que, en principio, pudiera parecer. Aunque la extranjerización puede, en ocasiones, respaldar, sin duda, ideologías minorizadas, sin embargo también puede, en otros casos, perjudicarlas notablemente bien porque no consiga trasladar el mensaje apropiado a los nuevos receptores (como podría ocurrir con una traducción extranjerizada del anuncio de Guinness) bien porque puede acabar defendiendo la normalización más canónica (véase el texto traducido de Bang \& Olufsen).

A fin de cuentas, el conocimiento de denotación, connotación, mito y contramito permite al traductor (y al consumidor) poner cierta distancia crítica frente a la seducción que ejerce el mensaje publicitario. Por una parte, la publicidad pretende colocar productos en el mercado para consolidar la situación de la élite canónica. Por otra, desata una vorágine consumista que se entiende como natural y lógica, y que aborta conatos de independencia de pensamiento.

No cabe duda de que la globalización y el consumismo asociado a ésta exhiben elevadas dosis de creatividad (término complejo que no definiremos aquí por motivos de espacio) para defender sus intereses. El reto que se nos plantea en el siglo XXI es el de favorecer prácticas desconstructivas para frenar esta novedosa forma de colonialismo. El traductor, como mediador multicultural, hará bien en describir y explicar la realidad que lo circunda para luego asumir un papel ético imprescindible.

\section{Bibliografía}

Aitchison, Jim (1999). Cutting Edge Advertising. How to Create the World's Best Print for Brands in the $21^{s t}$ Century. Singapore: Prentice Hall.

Al Shehari, Khalid (2001). The Semiotics and Translation of Advertising Texts: Conventions, Constraints and Translation Strategies with Particular Reference to English and Arabic. Unpublished Thesis. Manchester: UMIST.

Apter, Emily (2001). "On translation in the global market", Public Culture, 13(1). Online en: http://www.uchicago.edu/research/jnl-pub-cult/current/apter1.html. (consultado el 6 de enero de 2004).

Barthes, Roland (1972). Mythologies. Nueva York: Hilland Wang.

Barthes, Roland (1974). S/Z. Londres: Jonathan Cape.

Bassnett, Susan (1998). "The Translation Turn in Cultural Studies". S. Bassnett. \& A. Lefevere (eds) (1998). Constructing Cultures. Clevedon: Multilingual Matters, 123-140.

Beasley, Ron \& Danesi, Marcel (2002). Persuasive Signs. The Semiotics of Advertising. Berlín: Mouton de Gruyter. 
Beigbeder, Frédéric (2000). 13'99 euros. Barcelona: Anagrama.

Benedetti, Mario (1999). Inventario. Poesía 1950-1985. Madrid: Colección Visor.

Bueno, Antonio (2000). Publicidad y traducción. Soria: Vertere. Monográficos de la Revista Hermeneus, 2, Diputación de Soria.

Calzada Pérez, Maria (en prensa). "Selling Identities with the Help of Intertextuality. An Explanatory Framework for the Analysis of Original and Translated Advertisements". The Translator. Manchester: St. Jerome.

Chandler, Daniel (2001). Semiotics for Beginners. Online en: http://www.aberac.uk/media/Documents/S4B/sem01.html. (consultado el 6 de enero de 2004).

Chandler, Daniel (2002). Semiotics. The Basics. Londres/Nueva York. Routledge.

Cook, Guy (2001). The Discourse of Advertising. $2^{\mathrm{a}}$ edición. Londres/Nueva York: Routledge.

Corpas, Gloria et al. (eds). En torno a la traducción-adaptación del mensaje publicitario. Málaga: Universidad de Málaga, 13-48.

Elena García, Pilar (1990). Aspectos teóricos y prácticos de la traducción. AlemánEspañol. Salamanca: Universidad de Salamanca.

Elena García, Pilar (1994). Curso práctico de traducción general. Alemán-Español. Salamanca: Universidad de Salamanca.

Elena García, Pilar (2001). El traductor y el texto. Curso básico de traducción general (alemán-español). Barcelona: Ariel.

Genztler, Edwin (2002). "Translation, Poststructuralism and Power". M. Tymoczko \& E. Gentzler (eds). Translation and Power. Amherst/Boston: University of Massachussets Press, 195-218.

Goddard, Angela (1998). The Language of advertising. Londres/Nueva York: Routledge.

Gopegui, Belén (2001). Lo real. Barcelona: Anagrama.

Gorlee, Dinda (1994). Semiotics and the Problem of Translation. With Special Reference to the Semiotics of Charles S. Peirce. Amsterdam: Rodopi.

Hatim, Basil (1996). "The Method in their Adness: the Juggling of Texts, Discourse and Genres in the Language of Advertising and Implications for the Translator". A. Hurtado Albir (ed.). La enseñanza de la traducción. Castellón: Universidad Jaume I.

Hurtado Albir, Amparo (ed.). La enseñanza de la traducción. Castellón: Universidad Jaume I.

Klein, Naomi (2001). No logo. Barcelona: Paidós.

Martín, Jacinto et al. (1996). Los lenguajes especiales. Granada: Comares.

Osimo, Bruno (2001). Translation Course. Online en: http://www.logos.it/pls/dictionary/linguistic_resources.traduzione_en?lang=en.

Robinson, Douglas (1997). What is translation?. Kent: Kent State University Press.

Robinson, Mark (2000). The Sunday Times 100 Greatest Ads. London: Harper Collins.

Stam, Robert et al. (1992). New Vocabularies in Film Semiotics: Structuralism, PostStructuralism and Beyond. Londres: Routledge.

Tymoczko, Maria (1999). Translation in a Postcolonial Context. Manchester: St. Jerome.

Tymoczko, Maria \& Gentzler, Edwin (eds) (2002). Translation and Power. Amherst/Boston: University of Massachussets Press.

Vidal Claramonte, María del Carmen África (1998). El futuro de la traducción. Últimas teorías, nuevas aplicaciones. Valencia: Institució Alfons el Magnànim.

Vidal Claramonte, María del Carmen África \& Calzada Pérez, María (en prensa). "Translated Logos = Translated Identities? Connections between the 
Language of Advertising, Translation and Identity". Meta. Montreal: Les Presses de l'Université de Montréal.

Zambrana, Rafael (2002). "Ordenamiento jurídico de la publicidad en España". G. Corpas et al. (eds). En torno a la traducción-adaptación del mensaje publicitario. Málaga: Universidad de Málaga, 13-48. 